\title{
A Novel Microduplication in the Neurodevelopmental Gene SRGAP3 That Segregates with Psychotic Illness in the Family of a COS Proband
}

\author{
Nicole K. A. Wilson, ${ }^{1}$ Yohan Lee, ${ }^{1}$ Robert Long, ${ }^{1}$ Karen Hermetz, ${ }^{2}$ M. Katharine Rudd, ${ }^{2}$ \\ Rachel Miller, ${ }^{1}$ Judith L. Rapoport, $^{1}$ and Anjené M. Addington ${ }^{1}$ \\ ${ }^{1}$ Child Psychiatry Branch, National Institute of Mental Health, 10 Center Drive, Building 10/Room 3N202, Bethesda, \\ MD 20892, USA \\ ${ }^{2}$ Department of Human Genetics, Emory University School of Medicine, 615 Michael Street, Room 315, \\ Atlanta, GA 30322, USA
}

Correspondence should be addressed to Yohan Lee, leey2@mail.nih.gov

Received 22 June 2011; Accepted 14 July 2011

Academic Editors: C.-W. Cheng, E. Ergul, and D. Steinberger

Copyright ( 2011 Nicole K. A. Wilson et al. This is an open access article distributed under the Creative Commons Attribution License, which permits unrestricted use, distribution, and reproduction in any medium, provided the original work is properly cited.

Schizophrenia is a debilitating mental disorder affecting approximately $1 \%$ of the world's population. Childhood onset schizophrenia (COS), defined as onset before age 13, is a rare and severe form of the illness that may have more salient genetic influence. We identified a $\sim 134 \mathrm{~kb}$ duplication spanning exons $2-4$ of the Slit-Robo GTPase-activating protein 3 (SRGAP3) gene on chromosome 3 p25.3 that tracks with psychotic illness in the family of a COS proband. Cloning and sequencing of the duplication junction confirmed that the duplication is tandem, and analysis of the resulting mRNA transcript suggests that the duplication would result in a frame shift mutation. This is the first family report of a SRGAP3 copy number variant (CNV) in schizophrenia. Considering that SRGAP3 is important in neural development, we conclude that this SRGAP3 duplication may be an important factor contributing to the psychotic phenotype in this family.

\section{Introduction}

This paper documents a duplication in the neurodevelopmental gene Slit-Robo-GTPase activating protein 3 (SRGAP3; entrez gene ID: 9901) that segregates with psychotic illness in the family of a patient with childhood onset schizophrenia (COS). Schizophrenia is a severe and debilitating mental disorder characterized by hallucinations, delusions, flat and/or inappropriate affect, and cognitive impairment. It is a multifactorial disease, involving many unknown genetic as well as environmental factors. Although many potential genetic contributors have been identified, penetrance is generally below 20\% [1]. Childhood onset schizophrenia, defined as onset before age 13, is a rare and severe form of the illness that may be particularly informative for genetic studies [2-4].
High-density microarrays are a powerful tool for detecting submicroscopic chromosomal duplications and deletions with high resolution and efficiency [5]. COS probands have an increased rate of rare copy number variants $(\mathrm{CNVs})$ and a higher rate of other cytogenetic abnormalities [4]. A novel CNV of particular interest was an $\sim 134 \mathrm{~kb}$ duplication within the SRGAP3 gene on chr3p25.2-3p25.4 that was identified in a COS proband [4]. The duplication was transmitted from the father with schizotypal and avoidant personality disorders to the proband and his brother with schizophrenia. This is the first family report of a SRGAP3 CNV in schizophrenia and related disorders.

In healthy individuals, SRGAP3 is highly expressed in fetal and adult brain, and this difference is particularly striking in brain regions involved in higher cognitive function, learning, and memory [6]. SRGAP3 abnormalities 
have been observed at the genomic and expression level in several brain disorders including mental retardation [68], Parkinson's disease [9], and schizophrenia although previous schizophrenia reports did not have family data [10]. Additionally, the region of chromosome 3 containing SRGAP3 has been implicated in linkage studies of schizophrenia [11]. Here, we expand on our initial report of this discovery with sub-sequent cloning and sequencing of the duplication junction to investigate a $S R G A P 3$ duplication that segregates with psychotic illness in the family of a patient with childhood onset schizophrenia (COS).

\section{Case Presentation}

2.1. NSB 499. The proband, NSB499, walked and began speaking within normal limits but was placed in special education (no specific therapies) starting in the first grade primarily because of poor peer relationships dating back to his early childhood years. Starting around age 11, he became confused and disorganized and subsequently started to have auditory and visual hallucinations. He was hospitalized several times beginning around age 13 and his social and academic functioning deteriorated; he described "not being able to feel emotions anymore" and continued to hear voices telling him to hurt himself and others. The proband also experienced visual hallucinations of monster's faces, depressed feelings, anhedonia, sleep and appetite disturbances, and suicidal ideation. At age 14, the proband was admitted to our study and diagnosed with childhood onset schizophrenia, as well as general anxiety disorder, panic disorder, agoraphobia, and depression.

2.2. NSB 619. NSB619, the proband's older brother, also inherited the SRGAP3 duplication from the father. Although he too had normal developmental milestones, he was placed in special education (no specific therapies) in the first grade. NSB 619 experienced his first psychotic episode at age 17, and he was initially diagnosed with schizophrenia at age 18. The patient reported multiple delusions, visual hallucinations, and auditory hallucinations consisting of running commentary between voices conversing with each other. After schizophrenia onset, NSB 619 was incapacitated in his social functioning; he reported having no friends, experiencing discomfort in social situations, and lacking any activities that he enjoyed doing. During interview, it was noted that the patient had poor eye contact and inappropriate facial expressions and did not appear capable of comprehending interview questions.

2.3. NSB 617. The father, NSB 617 , reported being depressed as a child. He was held back in school twice, placed in special education, and did not graduate until he was 20 years old. Although he was married, at interview he reported having no close friends and experiencing discomfort in social situations. He also reported that he had not worked for years and spent most of his time watching TV. He exhibited impoverished speech, poor hygiene, constricted affect, and magical thinking and often made vague and irrelevant comments during interview. At interview he met criteria for schizotypal personality disorder, avoidant personality disorder, and a history of major depressive disorder.

2.4. Noncarrier Family Members. The mother of this family, NSB 618, was diagnosed with schizotypal and avoidant personality disorders as well as a history of major depressive disorder. The sister of the proband, NSB 622, was diagnosed with schizoid personality disorder although she was employed, married with two children, and acted normally in natural social situations. The eldest brother, NSB 621, was diagnosed with paranoid personality disorder. Finally, the second eldest brother, NSB 620, was placed in special education and held back in school twice.

\section{Results}

As part of whole-genome scans using Illumina $1 \mathrm{M}$ microarrays, we identified a $\sim 134 \mathrm{~kb}$ duplication in exons 24 of the Slit-Robo-GTPase activating protein 3 (SRGAP3) gene that is carried by the father and two schizophrenic brothers described above, but not by the mother or by the other three siblings. To characterize the location of the duplication, we performed fluorescence in situ hybridization (FISH) on metaphase chromosomes prepared from patientderived lymphoblastoid cell lines. Signals from fosmids corresponding to the duplication were observed at the ends of the short arms of chromosome 3. Signals were not observed on any other chromosomes, consistent with a local duplication (see Supplementary Figure 1 in Supplementary material available online at doi: 10.1155/2011/585893). The $\sim 134 \mathrm{~kb}$ duplication was too small to resolve by interphase FISH, thus, we determined the exact location of the duplication using high-resolution array comparative genome hybridization (CGH) and the orientation by using duplication junction cloning and breakpoint sequencing.

Microarray analysis revealed a gain of the short arm of chromosome 3 for the father and two sons from the same family. The minimum duplication coordinates in these three family members were chr3: 9,111,177-9,245,155 (Build 37, hg19) (Figure 1).

To determine the orientation of the duplication, we cloned the junction using a PCR strategy designed to capture either tandem or inverted duplications [12]. Sequencing the breakpoint junction PCR product revealed a $134 \mathrm{~kb}$ tandem duplication with the following coordinates: chr3: 9,111,132-9,245,356. We aligned the sequence of the tandem duplication breakpoint, which revealed 2 basepairs of microhomology at the junction (Supplementary Figure 2).

Sequencher analysis of the NM_014850.2 transcript of $S R G A P 3$ revealed that the tandem duplication would result in a shift of the mRNA reading frame, ultimately resulting 


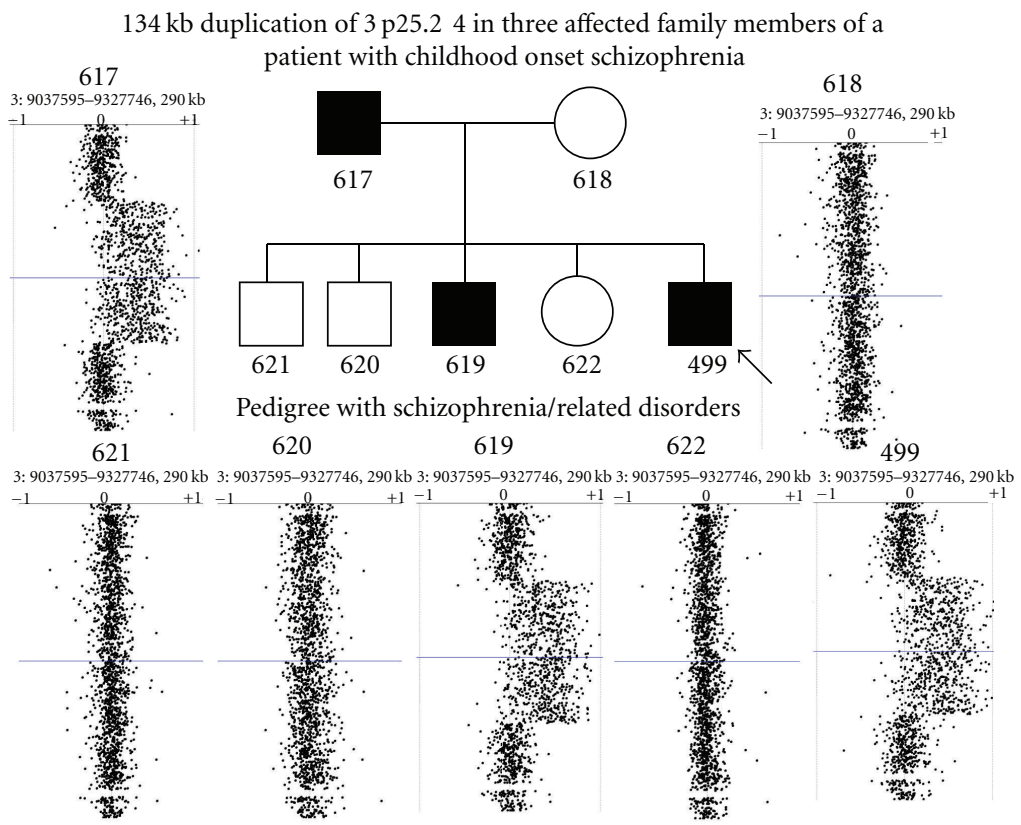

FIGURE 1: High-resolution microarray analysis of the family of a childhood onset schizophrenia proband. Results revealed a gain on the short arm of chromosome 3 for the proband (NSB499), his father (NSB617), and his brother (NSB619) with the minimum duplication coordinates of chromosome 3:9,111,177-9,245,155. Arrow on the pedigree indicates the COS proband. Clinical descriptions: 617: avoidant PD, schizotypal PD, history of major depressive disorder, held back in school twice. 618: avoidant PD, schizotypal PD, history of major depressive disorder. 621: paranoid PD. 620: held back in school twice. 619: schizophrenia. 622: schizoid PD. 499: childhood onset schizophrenia.

in six stop codons in the first 185 amino acids after the start of the duplication, with the first stop codon falling after the 73rd amino acid in the duplicated portion (Figure 2).

\section{Discussion}

This paper documents the first duplication in the neurodevelopmental gene SRGAP3 that segregates with psychotic illness in the family of a patient with childhood onset schizophrenia. This tandem duplication results in mRNA that undergoes a shift of the reading frame that would ultimately encode a stop codon, rendering this functionally a null mutation (Figure 2).

Schizophrenia is a multifactorial disease with multiple unknown genetic and environmental factors [13]. It is unlikely that any given case will be caused by a single mutation or that the phenotype resulting from any given mutation will be consistent $[1,13]$. One example of this is the widely studied schizophrenia susceptibility gene DISC1 that was first identified as a balanced translocation in a large Scottish family [14]. Of 77 family members studied, 34 carried the genetic abnormality [14]. Of the 34 carriers of the DISC1 genetic abnormality, 16 had various psychiatric diagnoses ranging from schizophrenia to alcoholism; there were also 5 of the 43 noncarrier family members who had a psychiatric diagnosis [14]. As with the pattern of penetrance observed in the family with a DISC1 translocation, we would not expect the SRGAP3 duplication to track perfectly with the psychotic phenotype. This SRGAP3 duplication is probably only one of many factors contributing to the psychotic phenotype in this family.

SRGAP3 abnormalities have been previously reported in several neurodevelopmental disorders [6-10]. Although noted without comment in a previous genome-wide assessment of CNVs in 1073 cases of schizophrenia and 1148 controls, two patients were identified with duplications in SRGAP3, and none of the controls had CNVs in this region although this did not reach significance and no family data was collected [10]. One of these duplications encompassed the same SRGAP3 exons that are duplicated in the family of NSB499 (Figure 3). Abnormalities in SRGAP3 have also been implicated in intellectual deficiency and Parkinson's disease [6-9].

A closer look at the molecular interactions of the SRGAP3 protein product reveals its potential relevance to neuronal development. The SRGAP3 protein product is known to enhance intrinsic activity of certain GTPases, particularly Rac1 and to a lesser extent CDC42 [6]. Interestingly, DISC1 has also been shown to regulate Racl activity, and constitutive Rac1 activation results in a decrease in the size of synaptic spines [15]. SRGAP3 may also regulate the size and density of dendritic spines by way of an inverse F-BAR domain that is characteristic of proteins from the SRGAP family [16]. This domain likely functions by utilizing a convex lipid-binding surface that facilitates the development of synaptic spines [16]. Furthermore, loss of SRGAP3 results 


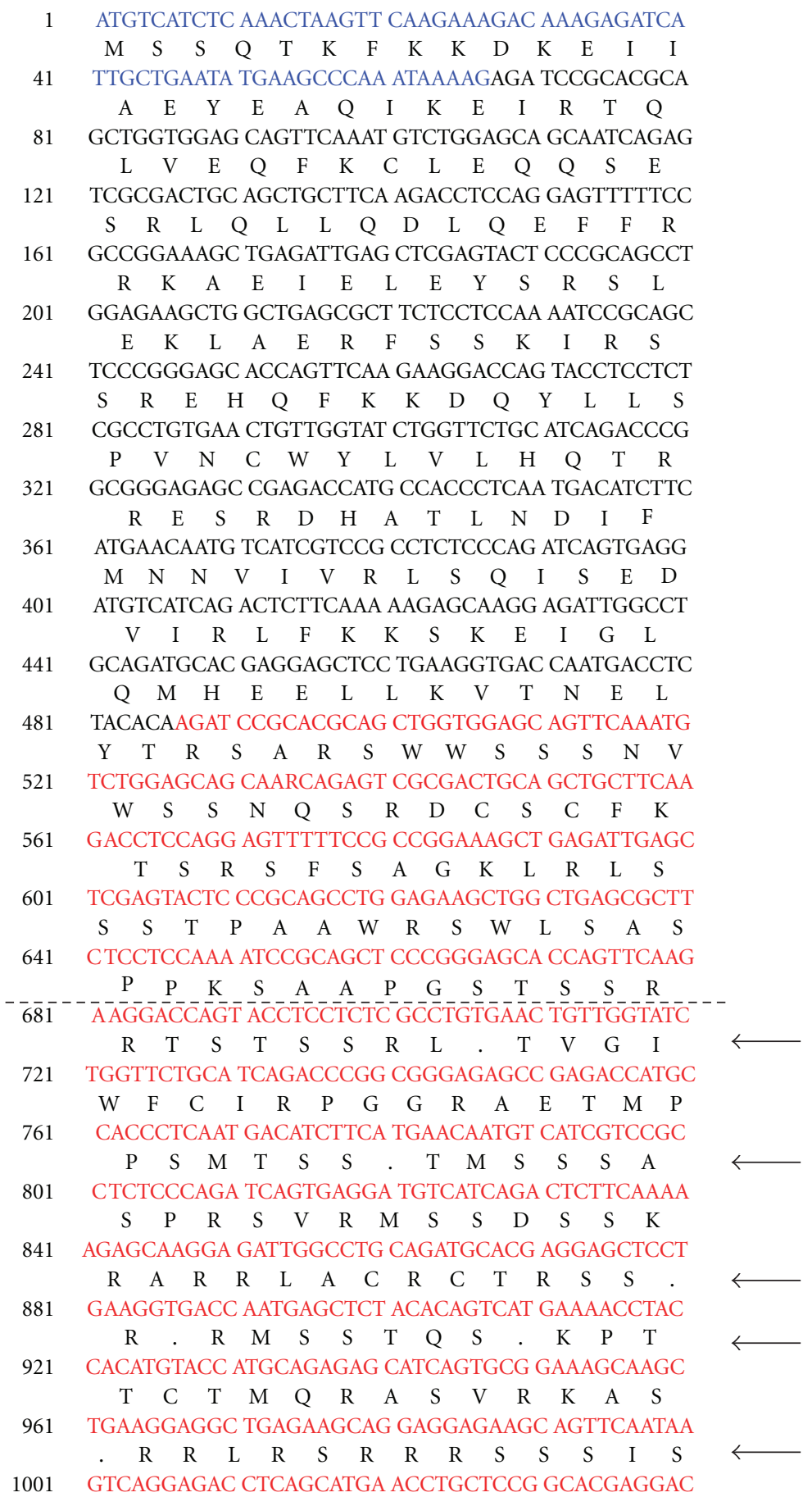

Figure 2: Predicted mRNA sequence and corresponding amino acid sequence generated using Sequencher 3.0. Exon 1 is shown in blue, and the tandem duplication of exons $2-4$ is shown in pink. The duplication causes a frameshift which leads to six stop codons in the first 185 amino acids after the start of the duplication (represented by dots; see arrows). These data suggest that the transcript would not be functional.

in a decrease in the density of dendritic spines both in vivo and in vitro [16].

Taken together, our findings and the studies cited above suggest that SRGAP3 may play an important role in neural development and that disruption or alteration of this gene may be partially etiologic for several mental disorders. The present study indicates that SRGAP3 may be a susceptibility gene for COS. Further investigation of association between SRGAP3 and schizophrenia is warranted. 


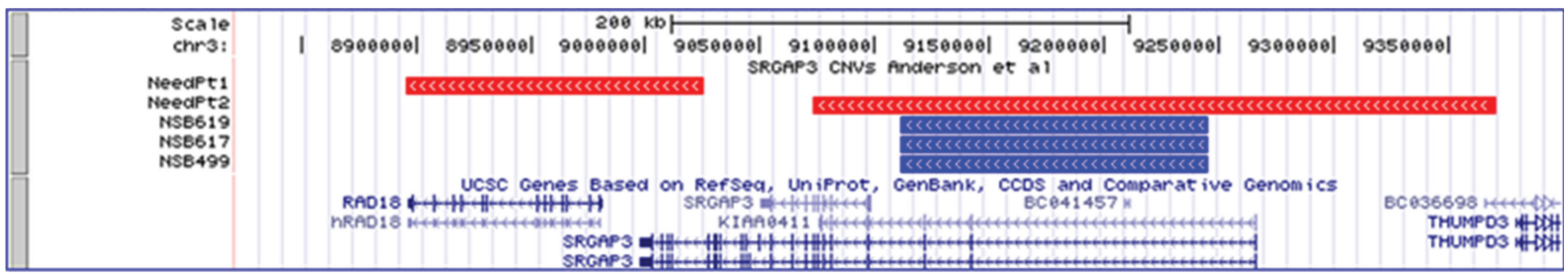

FIGURE 3: SRGAP3 duplications reported for a childhood onset schizophrenia proband (NSB499), his brother with schizophrenia (NSB617), and his father with other psychotic features (NSB619)(in blue, ascending order from bottom) aligned with SRGAP3 duplications previously seen in two adult patients [10] (shown in red).

\section{Acknowledgments}

The authors would like to thank Gale Germain, Julia Tossell, and Peter Gochman for helping with the clinical data. They would also like to thank Dede Greenstein for the help with the statistical analysis.

\section{References}

[1] E. Vassos, D. A. Collier, S. Holden et al., "Penetrance for copy number variants associated with schizophrenia," Human Molecular Genetics, vol. 19, no. 17, pp. 3477-3481, 2010.

[2] T. Walsh, J. M. McClellan, S. E. McCarthy et al., "Rare structural variants disrupt multiple genes in neurodevelopmental pathways in schizophrenia," Science, vol. 320, no. 5875, pp. 539-543, 2008.

[3] J. L. Rapoport, A. Addington, and S. Frangou, "The neurodevelopmental model of schizophrenia: what can very early onset cases tell us?" Current Psychiatry Reports, vol. 7, no. 2, pp. 8182, 2005.

[4] A. M. Addington and J. L. Rapoport, "The genetics of childhood-onset schizophrenia: when madness strikes the prepubescent," Current Psychiatry Reports, vol. 11, no. 2, pp. 156-161, 2009.

[5] G. Kirov, D. Gumus, W. Chen et al., "Comparative genome hybridization suggests a role for NRXN1 and APBA2 in schizophrenia," Human Molecular Genetics, vol. 17, no. 3, pp. 458-465, 2008.

[6] V. Endris, B. Wogatzky, U. Leimer et al., "The novel RhoGTPase activating gene MEGAP/ srGAP3 has a putative role in severe mental retardation," Proceedings of the National Academy of Sciences of the United States of America, vol. 99, no. 18, pp. 11754-11759, 2002.

[7] C. Gunnarsson and C. Foyn Bruun, "Molecular characterization and clinical features of a patient with an interstitial deletion of 3p25.3-p26.1," American Journal of Medical Genetics Part A, vol. 152, no. 12, pp. 3110-3114, 2010.

[8] S. Shuib, D. McMullan, E. Rattenberry et al., "Microarray based analysis of 3p25-p26 deletions (3p- syndrome)," American Journal of Medical Genetics Part A, vol. 149, no. 10, pp. 2099-2105, 2009.

[9] M. Elstner, C. M. Morris, K. Heim et al., "Single-cell expression profiling of dopaminergic neurons combined with association analysis identifies pyridoxal kinase as Parkinson's disease gene," Annals of Neurology, vol. 66, no. 6, pp. 792-798, 2009.

[10] A. C. Need, D. Ge, M. E. Weale et al., "A genome-wide investigation of SNPs and CNVs in schizophrenia," PLoS Genetics, vol. 5, no. 2, Article ID e1000373, 2009.
[11] S. G. Schwab, Irmansyah, Heriani et al., "Genome-wide scan in 124 Indonesian sib-pair families with schizophrenia reveals genome-wide significant linkage to a locus on chromosome 3p26-21," American Journal of Medical Genetics Part B, vol. 147, no. 7, pp. 1245-1252, 2008.

[12] M. F. Arlt, J. G. Mulle, V. M. Schaibley et al., "Replication stress induces genome-wide copy number changes in human cells that resemble polymorphic and pathogenic variants," American Journal of Human Genetics, vol. 84, no. 3, pp. 339-350, 2009.

[13] B. Mowry and D. Nancarrow, "Molecular genetics of schizophrenia," Clinical and Experimental Pharmacology and Physiology, vol. 28, no. 1-2, pp. 66-69, 2001.

[14] D. St Clair, D. Blackwood, W. Muir et al., "Association within a family of a balanced autosomal translocation with major mental illness," The Lancet, vol. 336, no. 8706, pp. 13-16, 1990.

[15] A. Hayashi-Takagi, M. Takaki, N. Graziane et al., "Disruptedin-Schizophrenia 1 (DISC1) regulates spines of the glutamate synapse via Rac1," Nature Neuroscience, vol. 13, no. 3, pp. 327-332, 2010.

[16] B. R. Carlson, K. E. Lloyd, and A. Kruszewski, "WRP/srGAP3 facilitates the initiation of spine development by an inverse F-BAR domain, and its loss impairs long-term memory," Journal of Neuroscience, vol. 31, no. 7, pp. 2447-2460, 2001. 


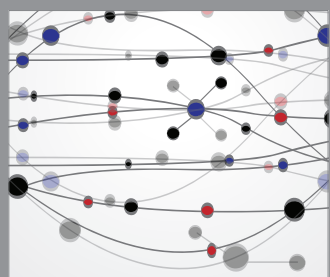

The Scientific World Journal
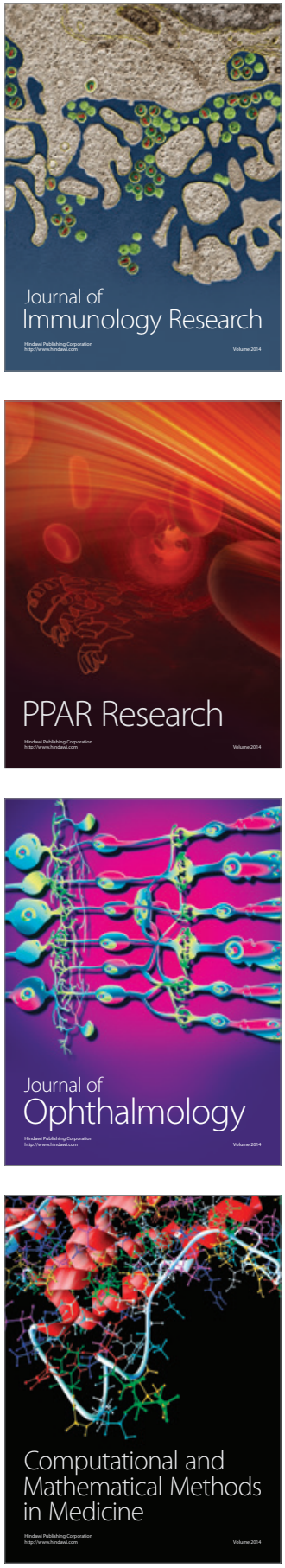

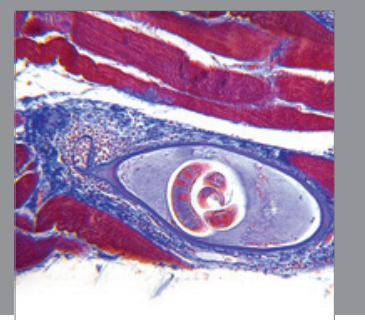

Gastroenterology

Research and Practice
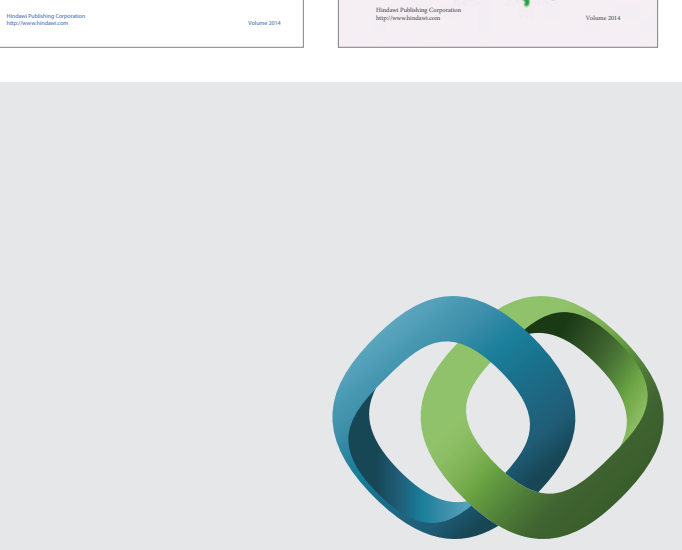

\section{Hindawi}

Submit your manuscripts at

http://www.hindawi.com
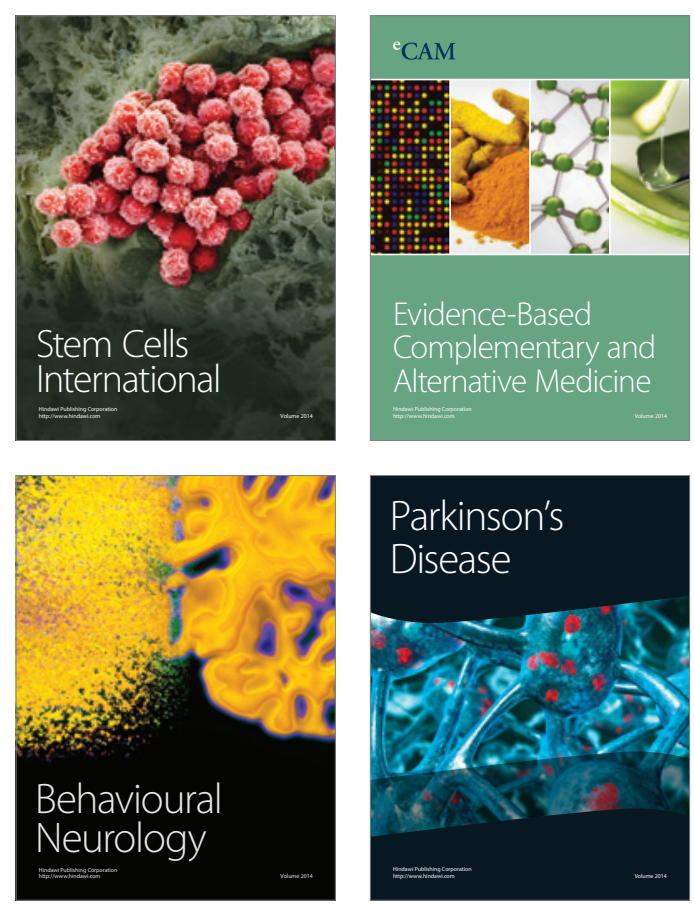

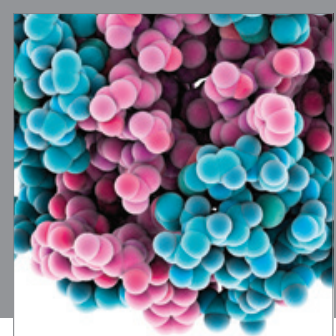

Journal of
Diabetes Research

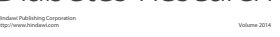

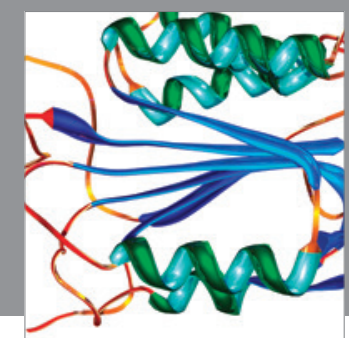

Disease Markers
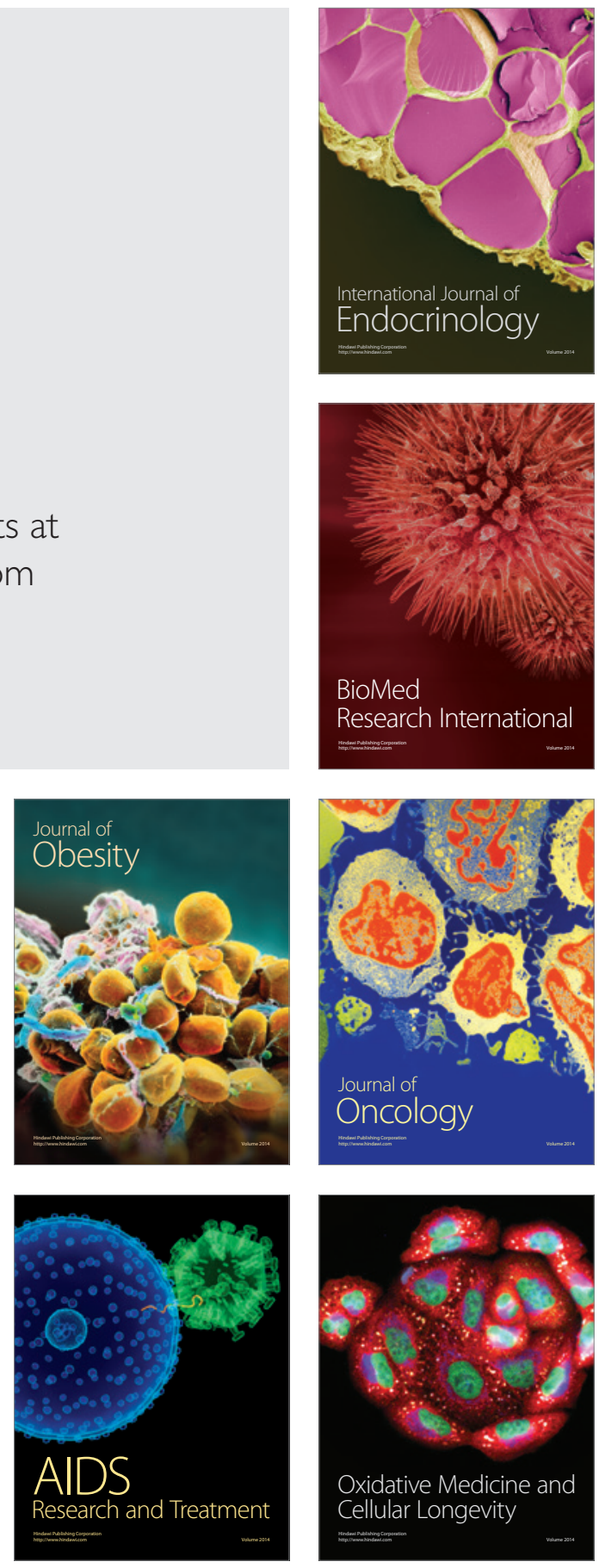